\title{
Eating Out: Fast Food Consumption and its Impact on Health of Young Generation of Karachi
}

\author{
Shaista Kamal Khan*, Dr. Khurram Khan Alwi**, Nimra Nadeem***
}

\begin{abstract}
This paper has measured the impacts of fast food on the health of the young generation. In today's age where both the genders are working as bread earners of the family, people prefer fast foods because of the convenience factor and eating fast food is affecting and influencing the bealth of the young generation. The core population, who consume fast food the most frequently is teenage youth. The study is based on value expectancy theory and attempts to develop and test a theory-based questionnaire that identifies factor relating to fast food consumption and impact on health. In this qualitative study, data is collected by interviews with seven persons. The predetermine open-ended questions had been made to investigate the thoughts of respondents and indicate the key meanings that each participant had described his or her experiences. After conducting interviews from participants then identified relevant themes and are further discussed in the results. All findings are supported by the literature review that is related to this study. Moreover, the qualitative study ends with some recommendations to the young generation.
\end{abstract}

Keywords: Fast food, Consumption, Health, Young Generation

JEL Classification: G21, M14

\section{INTRODUCTION}

The industry of fast-food has become one of the rapid-growing industries in the world in a very short period. Fast food, as per oxford dictionary definition "easily cooked processed and served in snack bars and restaurants as a quick meal or to be taken away" (Rasanthika \& Gunawardana, 2013; Verma \& Chawla, 2020). Unfortunately, fast food is the kind of food that is known as "junk food". Fast food is said to be rich in fats, Tran's fats, saturated fats, sodium and cholesterol and is hence, the main cause for several diseases prevalent nowadays. Fast food consumption can lead to illnesses and health problems like dietary deficiencies, cholesterol problems, diabetes, loss of muscle mass, heart diseases, cardiac disorders, sexual dysfunction, hypertension, skin allergies, depression, overweight, and obesity problems (Singh \& Mishra, 2014). The fast-food industry primarily began from Southern California in the 1940s altering the food consumption practices of individuals. Beginning as a trend from the American side, the fast-food industry has now become a multi-billion-dollar industry that has fascinated individuals from all over the world (Aysha \& Munazza, 2012; Verma \& Chawla, 2020). According to the UN Economic and Social Commission for Asia, 50\% of the world's population would be urban until 2020, half of which would be from Asia.

\footnotetext{
* Assistant professor, Department of Business Administration, Jinnah University for Women, Karachi,

Pakistan.Email: shaistakamalkhan@gmail.com (Corresponding Author)

**Associate professor, SZABIST Karachi,Pakistan.Email: Alwidrkhurramkhan@outlook.com

***Student, Department of Business Administration, Jinnah University for Women, Karachi, Pakistan. Email:

nimra.nadeem.24@gmail.com
} 
This is the reason that the fast-food organizations have been taking it as an opportunity to cater to the market segments of Asia, particularly India and Pakistan (Aysha \& Munazza, 2012). Along with the world, Pakistan's fast food industry is also changing remarkably as it is witnessing an inclined shift in the lifestyles, eating habits, and food choices of the people. Because of these reasons, fast food restaurants are becoming immensely popular among individuals of all ages in Pakistan. To mention a few, convenience, accessibility, better taste and affordability are some of the factors that have contributed significantly to the prevailing fast food consumption practices (Ebadi, Rakhshanderou, \& Ghaffari, 2018; Mamalis et al., 2019). Restaurants like KFC, McDonald's, Pizza Hut, Subway, Hardees, are all well-run own international chains that are working in Pakistan and hence, playing their part in the growth of the fast-food industry as well.

The fast-food industry in Pakistan is amongst the major industries in the country. Accounting for $27 \%$ of its value-added production and $16 \%$ of the total employment in the manufacturing sector, this industry caters to almost 180 million consumers in Pakistan. Pakistan is said to be the world's eighth-largest market when it comes to food and fast food-related industrial business (Aamir et al., 2018; Memon, 2016). The United Nations Economic and Social Commission for Asia predicted that until 2020, fifty per cent of the total world population would be urban out of which half would be from Asia. This is the reason that big fast food industrialists have been taking it as a chance to consider developing markets in Asian countries like Pakistan (Baig \& Saeed, 2012; Farooqui \& Alwi, 2019).

Purchasing and eating depend upon several factors such as economical, biological, cultural and psychological background and environment shape up the human food choices. Other factors such as convenience and monetary considerations have a secondary effect on the consumption choices (Agheli \& Emamgholipour, 2016; Yahya, Zafar, \& Shafiq, 2013). Moreover, the rise of obesity in the world is directly connected with the lack of physical fitness and health in both children and adults caused by the consumption of these detrimental foods. The Pakistan has one of the lowest prevalence of the physically active adult population in the world. This can be attributed to the changes in lifestyle, urbanization and modernization of the society leading individuals to alter their eating practices (Hashmi, Soomro, \& Saleem, 2013).

\section{Significance of Research}

This research provides information and awareness among the people about the physical and mental health issues in the young generation which is generating by the eating disorder. The main focus of this research paper is to aware the working parents, students and who are highly consuming fast food /junk food, especially those who have a high income or to maintain their high standards and because of easy excess or availability they are attracted towards the attractive deals but they are not aware that this fast food contains a high content of saturated fat, high salt, dietary fibre which cause the adverse effect of heart disease. This highlighted factor persuaded to conduct this research based on rising health issues. As the big fast-food tycoons aim their marketing and advertising strategies particularly towards, the oath. The fast-food industry tends to attract the youth masses by constantly inducing them to eat a staggering amount of fast food as a source of entertainment. Advertising is done primarily for children by attracting them towards children's meals along with toys and hence persuading them to consume more fast food (Nicklaus, 2020; Story \& French, 2004).

\section{Problem Statement}

The previous studies recommended that globalization has considerably affected the world as countries are adopting each other's lifestyles, food preferences, and culture due to this increasing trend of the fast-food culture, considered to be popular not only in the West but also in the Asian countries. Furthermore, the health of individuals is massively affected by their consumption which is yet another one of the biggest problems faced by individuals nowadays this paper will hence, also seek out the 
effects on the health of individuals that are caused due to increasing fast food consumption (Akhter, 2019; Aysha \& Munazza, 2012). One more study also suggested that due to an increasing trend towards junk food consumption has been introduced, the health issues are arising day by day as well and are receiving increasing attention in academics as well as in general researches (Sharif et al., 2017). Furthermore, earlier researches are suggested that should investigate the relationship between fastfood consumption, impact on health and why the outlets are increasing (Poelman et al., 2018). To identify gaps in the field of eating disorder and its effects on the health of the young generation. It is necessary to examine and search out more factors that can assist policymakers and health professionals to design satisfactory mediation. Furthermore, the studies can also target the point of view of parents, health professionals and policymakers and another contributor (Banik et al., 2020; Majabadi et al., 2016).

\section{Research Questions:}

This research is formulating the following questions:

frequently?

- What are the main determinants that are inducing people to consume fast food more

- What are the effects of fast food consumption on the health of individuals?

\section{Grounded Underpinning of Research Theory}

The current study is based on value expectancy theory (VET) that has been applied to describe certain health-related behavior. As indicated by Glantz refer to a person's belief of his or her capacity or perform in under a specific situation. It is a strong model for predicting either a person will take certain action within a particular situation. Value expectancy theory deal with the impact of individual values and expectation and values indicates the importance attached to the different life objectives while expectation refers to the perceived possibility of achievement of these objectives. The value and expectation are the strong motivation tools of behavior in the direction of achieving objectives (Ma, Castellanos, \& Bachman, 2016). Value expectancy theory is used to explore the influencing factors of fast-food consumption in the target population.

\section{LITERATURE REVIEW}

Several studies have been conducted to find and look out for the factors affecting the fastfood consumption among people. The literature on fast food consumption has declared that the negative impact on health and also shows the direct relationship between fast food consumption and health. Age of the individuals is said to shape up their dietary consumption choices. Young people are more likely to consume fast food as compared to their parents and older generations i.e., grandparents. Food consumption patterns before the last 3-4 decades, were entirely different. Since there was a less ratio of people eating out, most of the people consumed home-cooked food and were driven by their taste only. The childhood or adult obesity problems are considered to be complex in many countries because the availability of fast food increasing and adult are more image-conscious (Allman-Farinelli, Partridge, \& Roy, 2016; Braithwaite et al., 2014; Drewnowski \& Darmon, 2005).

Time and convenience are a very significant determinant of fast-food consumption according to the prior researches. As the male and female labour force participation in the modern world has risen, people generally look for foods that can save their time and be prepared easily and conveniently. In the past, research was also conducted where the emphasis was placed on convenience to be an important determinant of fast-food consumption because of spouse employment and the value of time (Jekanowski, Binkley, \& Eales, 2001; Okrent \& Kumcu, 2016; Yuan et al., 2019). 
Income is also considered to be an important cause for the rising trend of fast-food consumption in today's world. As people start earning more, they start spending more on food and hence, their practices of dining out increase. Although, fast food is available at affordable costs even for the middle- income earners, however, those with a higher spending power tend to try out different cuisines at different foods at elite and posh restaurants. This creates a demand for fast-food restaurants as the consumption level of the people increases (Janssen et al., 2018). In this context one more study is also explained, that fast food consumption becomes more common as the earning power increased along with the spending power of individuals also increased. The increased in incomes led to increased consumption of animal-based foods, high dense foods and processed foods and it shows a direct relationship with the increase in income level there is more purchasing or likeness toward fast food (Wang et al., 2020; Yahya et al., 2013). Food advertisements play a major role in this regard and have a direct relationship with fast food consumption along with exporting the culture, the United States is also exporting its food consumption and eating practices to individuals all over the globe. It, therefore, plays a very important role in shaping up the diet of people in many countries of the world (Verma \& Chawla, 2020).

The cost can be defined as the estimated price that is paid to buy or obtain something. In this context of the previous study, cost refers to the affordable prices of fast food that can easily be paid by individuals and fast food can hence, be consumed easily by middle and upper classes of our society. Apart from KFC, Mc Donald's, other local food chains are also operating within different countries that produce the same foods as big restaurants and attract people because of their low-pricing strategies. The same ingredients when used at home for the preparation of these foods were found out to be much higher than the restaurant cooked fast food (d'Amour et al., 2020; Kearney, 2010; Mills et al., 2017).

\section{RESEARCH METHODOLOGY}

\section{Research Design and Sample Size}

The qualitative research is applied where the researcher wants to gain more insight into individuals, this approach can be used (Corbin \& Strauss, 2014). When researcher contributes to the information based on constructivist standpoints and finds new experiences to create the pattern. The phenomenology method is selected in which emergent data is collected by open-ended questions with the evolving themes (Creswell \& Creswell, 2017). The rationale of the phenomenology method is suitable for this research because this method provides insight information about the phenomena of the study. The data is collected by open-ended questions through personal interviews. It allows the author to put himself itself in the place of that person and then recognize the personal encounters of individuals. In which the interviews and behaviors of people are studied for the data collection and for outcomes exploratory analysis are also added in this study which contain the finding of unique aspects that have never been judged earlier (Eisenhardt \& Graebner, 2007).

The methodology of this study is based on phenomenology, and various earlier studies are suggested different sample sizes, some studies are recommended sample size of qualitative study is 525 other suggested a minimum sample size is six. But ultimately essential sample size for data collection in qualitative study is based on point of saturation. Therefore, sample size of this study is seven according to the suggested criteria (Baxter \& Jack, 2008; Creswell \& Creswell, 2017; Guest, Bunce, \& Johnson, 2006). 


\section{Method of Data Collection}

This study was based on seven interviews transcripts that are a written record of all oral interview. The three interviews were conducted from the working women's, a gynecologist and an assistant professor at the hospital, one is working in a bank, and another is working in the university. The two interviews are taken by males one is working in a gym and another is nutritionist and dietitian working at the hospital. The Two respondents are university students. The interview was mainly based upon open-ended questions. The interviews were taken with all participants and written notes were made during the interview. Only three interviews were recorded as per permission given and other were not allowed. The interviews time with each participant was 40 to 50 minutes. All the participants freely volunteered to share their views and gave us very knowledgeable insights on the related topic. The research questions were carefully determined before the interview.

\section{RESULTS AND DISCUSSION}

The data transcripts from respondents were fully examined after completion of interviews, arranged and carefully reviewed for interpretation of data and to extract themes from the data. The transcripts were well-organized and seven themes were identified according to participant's perspectives (Braun \& Clarke, 2019). All the mentioned themes show how people health is affected in Pakistan by fast-food consumption and all mentioned below is the detailed explanation of the themes that were derived from the interviews.

\section{Food advertisements}

The interview conducted from the teenager, she also mentioned this aptly that she gets attracted from the promotional campaigns of fast-food restaurants on social media and the television. This way, she tries to avail these discounts and offer food from different food brands. Furthermore, working women (gynecologist) as the second respondent also mentioned that fast food promotions have a strong influence on the increase in consumption. The third one also indicates that food chains and restaurants operating worldwide using the communication medium of advertisements, instantaneous transfer of information and technological advancement, food consumption is said to be experiencing changes all over the world. Therefore, food advertisements play a major role in this regard (Henderson \& Kelly, 2005).

\section{Age}

The age is the strong determinant of over and unhealthy consumption in the young generation. The overall diet pattern of all age group is different, especially young adult are more inclined toward fast food. The age group 16 to 34 years old less likely to eat fruits and vegetables than adult age groups. However, once they grow up and live independently or after getting a job their family influence reduces, another factor such as time limitations, lack of food cooking skills, increase in income and reference group pressure may dominate on their food choices because they are immature according to age group. One more point, they do not know a healthy diet and what is the negative impact on health (Emond et al., 2019).

\section{Low Cost}

The affordability factor of fast foods has a direct impact on their consumption. People tend to consume fast food more as it is cheaper as compared to a full home-cooked meal. For example, as the participant stated burgers and pizzas when preparing at home are comparatively more expensive than the restaurant cooked fast food like KFC and Pizza Hut. The data finding also suggest, during the last 
10 years food consumption pattern drastically changed throughout the world. The low cost, tasty, speedy preparation are the main reason for fast-food consumption among the young generation. However, the price-value ratio is considered as an important factor in customer satisfaction and high consumption, also we can say low cost is the direct relation with food consumption (Zorbas et al., 2021).

\section{Time \& convenience}

The widely recognized definition of fast food is that is the type of food which prepared and served quickly in lesser time, usually supposed as an inexpensive meal. Fast foods take less time to get prepared and therefore are convenient for people with busy working schedules. Moreover, as answered by the working woman, since people at work don't have sufficient time to cook food at home, they opt for fast foods from restaurants, both for their children and themselves when at work. During the interview, the working woman said that she has long working hours and a very hectic working schedule. Hence, she comes home late and whenever her cook is not at home, she orders food for her children from restaurants or prepares foods herself from food brands like K\&Ns etc. that can easily and conveniently be prepared (Fulkerson, 2018).

\section{Income}

The interviews conducted from the respondents, they mentioned that the development all over the world has changed the lifestyle of people such as eating pattern and consumption pattern. The increase in revenue may increase the food consumption pattern particularly fast-food eating out. During the interview of working women indicates the food consumption is gone up due to more income when compared to lower or lesser income. The increase in income is not meaning the increase in people knowledge or rational thinking, the people are increasing intake of saturated fats and refined sugar that develop obesity and heart and skin problem (Fulkerson, 2018).

\section{Cultural Change}

The food culture of any area or region is indicated the health of population also culture dominate the eating pattern of the people. In the last decades all over the world, the food consumption pattern is going to change very rapidly due to globalization and advanced communication technology (Cheah et al., 2018). The ready to eat or fast food has become a very convenient and indispensable choice in modern society. In this context behavioral role played by the parents is very important. The economic development, globalization, urbanization and influence of European culture is the main determinant of fast-food consumption. If parents take their children to fast-food restaurants and order it for them at home, it means that they induce fast food consumption themselves in their children (Hashmi et al., 2013).

During the interview conducted from the nutritionist, she emphasized the same point. She stated that the attitude of parents as the major player in the development of this bad habit. Another reason the busy working schedule has a direct impact on the fast-food consumption of the people (Roudsari et al., 2017). People who work for longer hours usually tend to consume food that can easily be prepared and consumed to save time. This convenience factor that is derived from the busy schedules of the people, in this context, the nutritionist also added that in today's age where men and women are both working and have hectic work schedules, they often ignore the nutritional value of the foods and hence, tend to consume fast foods more (Farah \& Shahzad, 2020). Unfixed and improper meal times also put an impact on the consumption of fast foods. Employed people and students, in particular, have very hectic working schedules and therefore, they neglect their diet very frequently. The teenager, who is also a student shared the same thoughts. During the interview, she said that because of her university timings, she often misses her lunch and opts for a burger or a 
sandwich from her university cafeteria. This way fast food consumption is triggered whilst decreasing healthy meals consumption.

\section{Health}

After talking interview with the nutritionist, analyzed that the fast-food consumption has a direct impact on the health of individuals and she said poor diet are increasing the risk factor of mortality and illness globally. Fast foods are rich in fats containing high levels of refined sugars, slat a numerous food additive such as monosodium glutamate and tartrazine, lacking all the essential healthy nutrients at the same time. All these can cumulatively lead to many detrimental diseases and illnesses like dietary deficiencies, cholesterol problems, diabetes, loss of muscle mass, heart diseases, cardiac disorders, sexual dysfunction, hypertension, skin allergies, depression, overweight and obesity (Farah \& Shahzad, 2020). Furthermore, other two respondents also highlight the issues of health after fastfood consumption and said most of the peoples likes young generation neglect their health because of the little knowledge that they pursue about their food consumption practices. They ignore the bad effects of fast food on their health and continue with their consumption. Therefore, this has a direct influence on the increasing eating of fast food among people, especially young adult and children (Farah \& Shahzad, 2020)

\section{CONCLUSION}

The findings based on literature and all the information gathered through interviews show that all themes play a significant role in the determination of fast-food consumption. The global acceptance of fast food has a strong influence on our society. People are increasing dining out; particularly the young generation is gradually motivated toward fast food. The reason why fast food is consumed by individuals is very well determined from the identification of the themes People have increased their consumption that has triggered the birth of many detrimental and lethal diseases. Therefore, people must lookout for ways to change their lifestyles, eating habits and make intelligent diet decisions, considering the health and nutritional value of whatever foods they consume.

In a world where awareness regarding everything is so easy to reach people, they must take out some time for themselves and search more about how to look after their health. They must stay away from foods that can affect their health in the long run and rather opt for foods that have vitamins, proteins and are rich in nutrients. This research is helpful to resolve the health issues, which are growing day by day in all over the world. This study will indicate how to prevent from diseases, which can take place by taking harmful food so that how people could take care of their health, and give them a happy long life. This study also is going to help people get a better understanding of the factors that determine the increasing consumption of fast food. Moreover, this study also attempts to throw light on the effects of fast-food consumption on the health of individuals. The causes and effects of the fast-food consumption will hence be considered in detail in this research paper.

\section{Recommendations}

The recommendations based on the findings and the themes that were extracted. First and the foremost thing is that people should look after their health. It is a very common quotation that, 'health is wealth'. Health is, therefore, undoubtedly the wealth of an individual and must wholly be taken care of. Individuals should monitor their diet, eating habits and look for where they can change their meal. For example, instead of consuming excess carbohydrates and unneeded fats in our diet, we must opt for foods that are rich in nutrients and vitamins and are good for our health. Nowadays, people often neglect their diet because of time. They don't have time to cook food at home due to their busy 
working routines and hence, ignore their health. People should, therefore, learn how to manage their time effectively so that neither their work nor health gets affected. Rather than opting for unhealthy foods, people must consider the intake of fruits and vegetables over the consumption of fatty acids that only tend to make people lazier and obese.

Parents must also take steps to minimize the consumption of fast foods in their children. Instead of giving them these kinds of foods for lunch, they must give their children fruits and healthy home-cooked meals for lunch. Also, to set good examples for their children, they must model healthy eating and avoid junk foods themselves first. Moreover, the management of all schools must monitor the eating habits of their students and provide them with a healthy lunch timetable that should hence, be followed by all students. Soft drinks usage in schools should strictly be prohibited.

Health education along with the proper implementation of a healthy diet can improve the dietary habits of children. Laws must be made for the regulation of marketing and selling fast food that is responsible for inducing the eating of fast food in children. Such steps can help in controlling the eating of fast food. In the last but not least, we would recommend the people to educate themselves about their diet. If people are aware and educated about what they eat, how many calories they consume and have the proper knowledge and know-how of the nutritional value of their foods, they will eventually be inclined towards healthy eating and as a result, ditch their unhealthy eating habits. Amidst their busy and work loaded lifestyles, people shouldn't neglect their health-related issues and look after themselves in a good way.

\section{REFERENCES}

Aamir, M., Ahmad, H., Javaid, Q., \& Hasan, S. M. (2018). Waste not, want not: a case study on food waste in restaurants of Lahore, Pakistan. Journal of Food Products Marketing, 24(5), 591-610.

Agheli, L., \& Emamgholipour, S. (2016). Analyzing fast food consumption among Iranian urban households. International Review of Management and Marketing, 6(2), 205-212.

Akhter, M. M. (2019). Consumer Attitude toward Fast Food Consumption: A Study on University Students in Dhaka City. Asian Journal of Humanity, Art and Literature, 6(1), 21-32.

Allman-Farinelli, M., Partridge, S. R., \& Roy, R. (2016). Weight-related dietary behaviors in young adults. Current obesity reports, 5(1), 23-29.

Aysha, K., \& Munazza, S. (2012). Review of Trends in Fast Food Consumption. European Journal of Economics Finance and Administrative Sciences ISSN, 1450-2275.

Baig, A. K., \& Saeed, M. (2012). Review of trends in fast food consumption. European Journal of Economics, Finance and Administrative Sciences, 48, 77-85.

Banik, R., Naher, S., Pervez, S., \& Hossain, M. M. (2020). Fast food consumption and obesity among urban college going adolescents in Bangladesh: a cross-sectional study. Obesity Medicine, 17, 100161.

Baxter, P., \& Jack, S. (2008). Qualitative case study methodology: Study design and implementation for novice researchers. The qualitative report, 13(4), 544-559.

Braithwaite, I., Stewart, A. W., Hancox, R. J., Beasley, R., Murphy, R., Mitchell, E. A., \& Group, I. P. T. S. (2014). Fast-food consumption and body mass index in children and adolescents: an international cross-sectional study. BMJ open, 4(12), e005813. 
Braun, V., \& Clarke, V. (2019). Reflecting on reflexive thematic analysis. Qualitative Research in Sport, Exercise and Health, 11(4), 589-597.

Cheah, J. H., Sarstedt, M., Ringle, C. M., Ramayah, T., \& Ting, H. (2018). Convergent validity assessment of formatively measured constructs in PLS-SEM: On using single-item versus multiitem measures in redundancy analyses. International Journal of Contemporary Hospitality Management.

Corbin, J., \& Strauss, A. (2014). Basics of qualitative research: Techniques and procedures for developing grounded theory. Sage publications.

Creswell, J. W., \& Creswell, J. D. (2017). Research design: Qualitative, quantitative, and mixed methods approaches. Sage publications.

d'Amour, C. B., Pandey, B., Reba, M., Ahmad, S., Creutzig, F., \& Seto, K. C. (2020). Urbanization, processed foods, and eating out in India. Global Food Security, 25, 100361.

Drewnowski, A., \& Darmon, N. (2005). Food choices and diet costs: an economic analysis. The Journal of nutrition, 135(4), 900-904.

Ebadi, L., Rakhshanderou, S., \& Ghaffari, M. (2018). Determinants of fast food consumption among students of Tehran: Application of planned behavior theory. International Journal of Pediatrics, 6(10), 8307-8316.

Eisenhardt, K. M., \& Graebner, M. E. (2007). Theory building from cases: Opportunities and challenges. Academy of management journal, 50(1), 25-32.

Emond, J. A., Longacre, M. R., Drake, K. M., Titus, L. J., Hendricks, K., MacKenzie, T., ... \& Dalton, M. A. (2019). Influence of child-targeted fast food TV advertising exposure on fast food intake: A longitudinal study of preschool-age children. Appetite, 140, 134-141.

Farah, M. F., \& Shahzad, M. F. (2020). Fast-food addiction and anti-consumption behaviour: The moderating role of consumer social responsibility. International Journal of Consumer Studies, 44(4), 379-392.

Farooqui, M., \& Alwi, S. K. K. (2019). Fast Food Trend Analysis by Evaluating Factors Leading to Customer Satisfaction. Journal of Marketing and Consumer Research, 55, 55-66.

Fulkerson, J. A. (2018). Fast food in the diet: Implications and solutions for families. Physiology \& behavior, 193, 252-256.

Guest, G., Bunce, A., \& Johnson, L. (2006). How many interviews are enough? An experiment with data saturation and variability. Field methods, 18(1), 59-82.

Hashmi, A., Soomro, J. A., \& Saleem, K. (2013). Food behaviors and youth obesity in Pakistan. Acta Scientiarum. Health Sciences, 35(2), 223-229.

Henderson, V. R., \& Kelly, B. (2005). Food advertising in the age of obesity: content analysis of food advertising on general market and African American television. Journal of nutrition education and behavior, 37(4), 191-196. 
Janssen, H. G., Davies, I. G., Richardson, L. D., \& Stevenson, L. (2018). Determinants of takeaway and fast food consumption: a narrative review. Nutrition research reviews, 31(1), 16-34.

Jekanowski, M. D., Binkley, J. K., \& Eales, J. (2001). Convenience, accessibility, and the demand for fast food. Journal of Agricultural and Resource Economics, 58-74.

Kearney, J. (2010). Food consumption trends and drivers. Philosophical transactions of the royal society B: biological sciences, 365(1554), 2793-2807.

Ma, R., Castellanos, D. C., \& Bachman, J. (2016). Identifying factors associated with fast food consumption among adolescents in Beijing China using a theory-based approach. Public bealth, 136, 87-93.

Majabadi, H. A., Solhi, M., Montazeri, A., Shojaeizadeh, D., Nejat, S., Farahani, F. K., \& Djazayeri, A. (2016). Factors influencing fast-food consumption among adolescents in tehran: a qualitative study. Iranian Red Crescent Medical Journal, 18(3).

Mamalis, S., Mylona, I., Kamenidou, I. E., \& Pavlidis, S. (2020). Exploring Consumers' Orientation Toward Fast Food Consumption in Greece. Springer Proceedings in Business and Economics, 373-383.

Memon, N. A. (2016). Fast food: 2nd largest industry in Pakistan. Pakistan Food Journal, 3(1), 1-3.

Mills, S., White, M., Brown, H., Wrieden, W., Kwasnicka, D., Halligan, J., ... \& Adams, J. (2017). Health and social determinants and outcomes of home cooking: A systematic review of observational studies. Appetite, 111, 116-134.

Nicklaus, S. (2020). Eating and Drinking in Childhood. Handbook of Eating and Drinking: Interdisciplinary Perspectives, 391-412.

Okrent, A. M., \& Kumcu, A. (2016). US households' demand for convenience foods (No. 1477-2017-3961).

Poelman, M., Strak, M., Schmitz, O., Hoek, G., Karssenberg, D., Helbich, M., ... \& Vaartjes, I. (2018).

Relations between the residential fast-food environment and the individual risk of cardiovascular diseases in The Netherlands: A nationwide follow-up study. European journal of preventive cardiology, 25(13), 1397-1405.

Rasanthika, A. H. B. S., \& Gunawardana, T. S. L. W. (2013, February). Fast food consumption: identifying working women's attitudes towards consumption of fast food. In Proceedings of the International Conference on Mnagement and Economics (pp. 1-10).

Roudsari, A. H., Vedadhir, A., Amiri, P., Kalantari, N., Omidvar, N., Eini-Zinab, H., \& Sadati, S. M. H. (2017). Psycho-socio-cultural determinants of food choice: A qualitative study on adults in social and cultural context of Iran. Iranian journal of psychiatry, 12(4), 241.

Sharif, M. Z., Alcalá, H. E., Albert, S. L., \& Fischer, H. (2017). Deconstructing family meals: Do family structure, gender and employment status influence the odds of having a family meal?. Appetite, 114, 187-193.

Singh, M., \& Mishra, S. (2014). Fast food consumption pattern and obesity among school going (9-13 year) in Lucknow District. International Journal of Science and Research, 3(6), 1672-4. 
Story, M., \& French, S. (2004). Food advertising and marketing directed at children and adolescents in the US. International Journal of Behavioral Nutrition and Physical Activity, 1(1), 1-17.

Verma, S., \& Chawla, G. K. (2020). Convenience Food: An Emerging Trend in India. EXECUTIVE EDITOR, 11(9), 173-176.

Wang, B., Liu, L., Qiao, D., Xue, Y., Liu, X., Zhang, D., ... \& Li, X. (2020). The association between frequency of away-from home meals and type 2 diabetes mellitus in rural Chinese adults: the Henan Rural Cohort Study. European journal of nutrition, 59(8),1-11.

Yahya, F., Zafar, R., \& Shafiq, S. (2013). Trend of fast food consumption and its effect on Pakistani society. Food Science and Quality Management, 11, 1-7.

Yuan, M., Seale Jr, J. L., Wahl, T., \& Bai, J. (2019). The changing dietary patterns and health issues in China. China Agricultural Economic Review.

Zorbas, C., Lee, A., Peeters, A., Lewis, M., Landrigan, T., \& Backholer, K. (2021). Streamlined datagathering techniques to estimate the price and affordability of healthy and unhealthy diets under different pricing scenarios. Public Health Nutrition, 24(1), 1-11. 